A. Lundby (second from left) waits while E.G. Michaelis, who was Scientific Secretary of the Conference, signs for his volume of the Proceedings. Distributing the copies are H. Coblans, head of the Scientific Information Service, and Rhona Ball, his secretary.

On 25 October the publication in record time of the Proceedings of the 1962 International conference on high-energy physics ivas celebrated at a small party given by the Director-general.

Copies of the book were available for those who had ordered them only fifteen weeks after the end of the conference. Edited by $J$ J Prentki, with an editorial committee of $R$. Armenteros, $V$. Glaser and A.E. Taylor, the Proceedings were published by the Scientific Information Service of CERN (from ivhom copies may be obtained at the low price of $\mathbf{6 0} \mathbf{S} \mathbf{w}$ is $\mathbf{f r a n c s )}$.

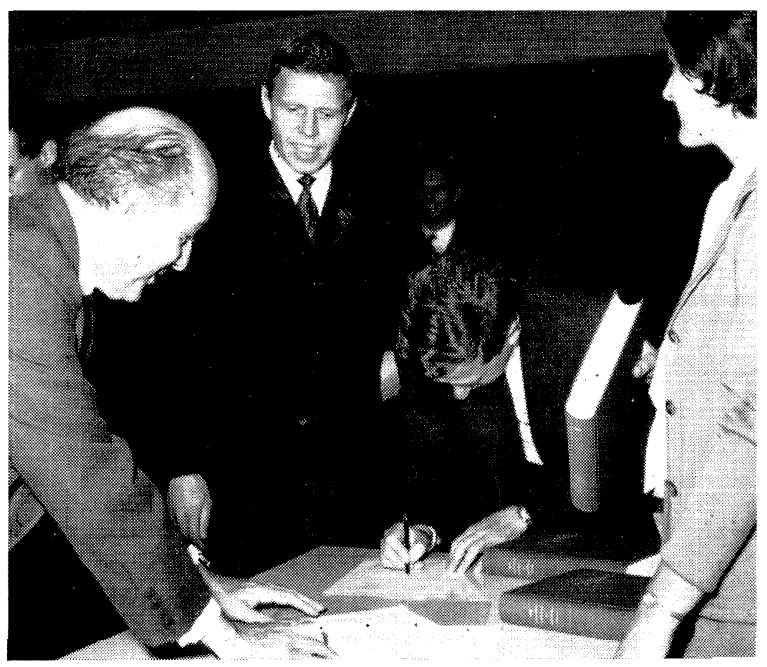

\title{
Conference Proceedings Published
}

We print beloiv a review of the volume by Prof. H. Feshbach,
has recently arrived at CERN to spend a year with the Theory Division.

This was undoubtedly a most exciting conference. To be sure a sizeable fraction of the world's most able physicists were here at CERN. But more important they had many things to report, much to discuss and clarify. And there was the realization, sometimes explicitly recognized, that a new branch of physics had come of age. For the first time there was confidence that the various landmarks and geographical features of this new world, which had been slowly making their appearance, would all come completely into view and that it would shortly be possible to ask the more subtle dynamical questions dealing with its topographical structure. And one must remember that fifteen years ago there were only some vague intimations of its existence ! The entire evolutionary process has been compressed into this short period, and because of this we have a remarkably clear-cut example of the operation and power of the 'scientific method'. It may sound trite to say so, but this writer has found it most thrilling to watch the experimental information develop from very vague first indications and the theories from speculative analogies.

The 'Proceedings' follow the general plan of the conference. There were several sessions running in parallel in which various invited as well as contributed papers were delivered. These were supposedly summarized in plenary sessions for the conference as a whole by the 'rapporteurs'. Not all of these gentlemen were faithful to their tasks. Several of the rapporteurs articles are superb but for others it is necessary to

Each copy of the Proceedings

- weighs $2.5 \mathrm{~kg}$

- measures $28 \mathrm{~cm} \times 23 \mathrm{~cm} \times 5 \mathrm{~cm}$

- contains 976 pages

- records 208 lectures and the discussion of 20 parallel, 1 special and 13 plenary sessions

- contains 810 illustrations, with 2 colour plates

- lists the names and addresses of 357 scientists from outside CERN who attended the conference

- indexes the names of 764 scientists who were part authors of papers or took part in the discussions. refer back to the records of the original sessions in order to recover the available information fully. Be that as it may, reading the rapporteurs' papers and the summary delivered by V.F. Weisskopf will not only provide a framework for the more detailed sessions but will also reveal what were considered to be the most significant developments.

A special session was devoted to the neutrino ex periment in which it was demonstrated that the neutrino emitted in pi-mu decay differs from that emitted in nuclear beta-decay. The cross-sections involved here are of the order of $10 \sim^{38} \mathrm{~cm}^{2}$, which points up the great advances in technique which must have been made in order to make it possible to observe these very rare events. Such selectivity, it might be added, is necessarily expensive. It is amusing to recall that one of the goals of the original meson theory of nuclear forces was to explain nuclear beta-decay; it turned out that the experimental particle on which this notion was based was not the pi-meson, and to add insult to injury the neutrino emitted in pi-decay bears no simple relation to the beta-decay neutrino.

Although this experiment resolves one of the contradictions in the theory of weak interactions a very important 'difficulty' remains. The word difficulty is put in quotes only to indicate that is exists only within the framework of a certain set of hypotheses on weak interactions, namely the universal current-current coupling scheme. If this hypothesis is wrong the difficulty disappears. However, assuming its validity the experimental measurements showing that strangeness can change by two units in weak decays are in contradiction with the ratio of the decay probabilities to the mass difference of the $\mathrm{K}^{\circ} \mathrm{i}$ and $\mathrm{K}^{\circ} \mathbf{2}$ particles.

But these fascinating weak-interaction problems are really only tangential to the developments alluded to at the beginning of this review. For these we must turn to the strong-interaction sessions in which the meson (pi and $\mathrm{K}$ ) interaction with baryons, the pion-pion and the pion-kaon interactions were discussed. There is much new data. There are the newly discovered multi-pion systems such as the omega, rho and eta particles. There are new baryon resonances induced by the mesonbaryon interaction. There is much structure and we can expect more. But most remarkably there are theoretical 